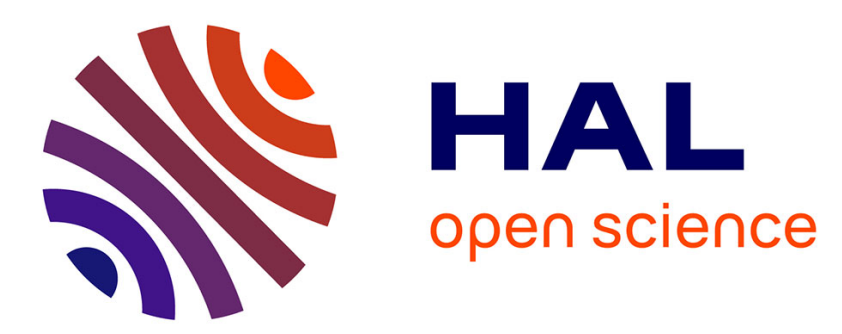

\title{
A local projection stabilization of fictitious domain method for elliptic boundary value problems
}

\author{
Amdouni Saber, Maher Moakher, Yves Renard
}

\section{To cite this version:}

Amdouni Saber, Maher Moakher, Yves Renard. A local projection stabilization of fictitious domain method for elliptic boundary value problems. Applied Numerical Mathematics, 2014, 76, pp.60-75. hal-00713115

\section{HAL Id: hal-00713115 \\ https://hal.science/hal-00713115}

Submitted on 29 Jun 2012

HAL is a multi-disciplinary open access archive for the deposit and dissemination of scientific research documents, whether they are published or not. The documents may come from teaching and research institutions in France or abroad, or from public or private research centers.
L'archive ouverte pluridisciplinaire $\mathbf{H A L}$, est destinée au dépôt et à la diffusion de documents scientifiques de niveau recherche, publiés ou non, émanant des établissements d'enseignement et de recherche français ou étrangers, des laboratoires publics ou privés. 


\title{
A local projection stabilization of fictitious domain method for elliptic boundary value problems
}

\author{
S. Amdouni ${ }^{1}$, M. Moakher ${ }^{2}$, Yves Renard ${ }^{3}$.
}

\begin{abstract}
In this paper, a new consistent method based on local projections for the stabilization of a Dirichlet condition is presented in the framework of finite element method with a fictitious domain approach. The presentation is made on the Poisson problem but the theoretical and numerical results can be straightforwardly extended to any elliptic boundary value problem. A numerical comparison is performed with the Barbosa-Hughes stabilization technique. The advantage of the new stabilization technique is to affect only the equation on multipliers and thus to be equation independent.
\end{abstract}

Keywords: finite element method, Xfem, fictitious domain method, Poisson problem.

\section{Introduction}

The fictitious domain method is a technique allowing the use of regular structured meshes on a simple shaped fictitious domain containing the real domain. Generally, this technique is used for solving elliptic problems in domains with unknown or moving boundary without having to build a body fitted mesh. There exist two main approaches of fictitious domain method. The "thin" interface approach where the approached interface has the same dimension as the original interface. This approach was initiated by V.K. Saul'ev in [24]. In this context, there exist different techniques to take account of the boundary condition: the technique where the fictitious domain mesh is modified locally to take account of the boundary condition (see for instance reference $[24,15])$, The technique of penalization which allows to conserve the Cartesian mesh of the fictitious domain (see for instance reference $[2,12]$ ) and the technique of Lagrange multiplier introduced by R. Glowinski et al. $[9,10,12,11]$ where a second mesh is considered to conserve the Cartesian mesh of the fictitious domain and to take account of the boundary condition.

The second approach of fictitious domain method is the "Spread" interface approach where the approximate interface is larger than the physical interface. The approximate interface has one dimension more than the original one. It was introduced by Rukhovets [23]. For example, the following methods can be found in this group: Immersed boundary method [20, 21] and Fat boundary method $[16,6]$.

Recently, fictitious domain methods with a thin interface have been proposed in the context of the extended finite element method (X-FEM) introduced by Moes, Dolbow and Belytscko [18]. Different approaches are proposed in $[17,26,3]$ to directly enforce an inf-sup condition on a multiplier to prescribe a Dirichlet boundary condition. Another possibility is the use of the stabilized Nitsche's method [19] which is close to a penalization technique but is consistent and avoids large penalty terms that would otherwise deteriorate the conditioning of the system matrix

\footnotetext{
${ }^{1}$ Laboratoire LAMSIN, Ecole Nationale d'Ingénieurs de Tunis, Université Tunis El Manar, B.P. 37, 1002 TunisBelvédère, Tunisie 6 INSA-Lyon, ICJ UMR5208-France. Saber.Amdouni@insa-lyon.fr.

${ }^{2}$ Laboratoire LAMSIN, Ecole Nationale d'Ingénieurs de Tunis, Université Tunis El Manar, B.P. 37, 1002 TunisBelvédère, Tunisie. maher.moakher@enit.rnu.tn.

${ }^{3}$ Université de Lyon, CNRS, INSA-Lyon, ICJ UMR5208, LaMCoS UMR5259, F-69621, Villeurbanne, France. Yves.Renard@insa-lyon.fr.
} 
[8]. We can cite also the method introduced in [7] which uses a stabilized Lagrange multipliers method using piecewise constant multipliers and an additional stabilization term employing the inter-element jumps of the multipliers. Finally let us mention the work of Haslinger and Renard [13] where an a priori error estimate for non-stabilized Dirichlet problem is given and an optimal method is developped with the use a Barbosa-Hughes stabilization (see $[4,5])$.

In this paper, we perform a study similar to [13] for a new stabilization technique applied to the fictitious domain method inspired by the X-FEM. The principle of this new stabilization technique is to penalize the difference of the multiplier with its projection on some pre-defined patches. The advantage of this method is of at least threefold: the method is fully consistent, there is no use of mesh other than the (possibly cartesian) one of the fictitious domain and the additional term concerns only the multiplier and is not model dependent such as the BarbosaHughes stabilization technique.

The paper is organised as follows. In Section 1 we introduce the Poisson model problem and in Section 2, the non-stabilized fictitious domain method. We present our new stabilization technique in Section 3 together with the theoretical convergence analysis. Finally, Section 4 is devoted to two and three-dimensional numerical experiments and the comparison with the use of Barbosa-Hughes stabilization technique.

\section{The model problem}

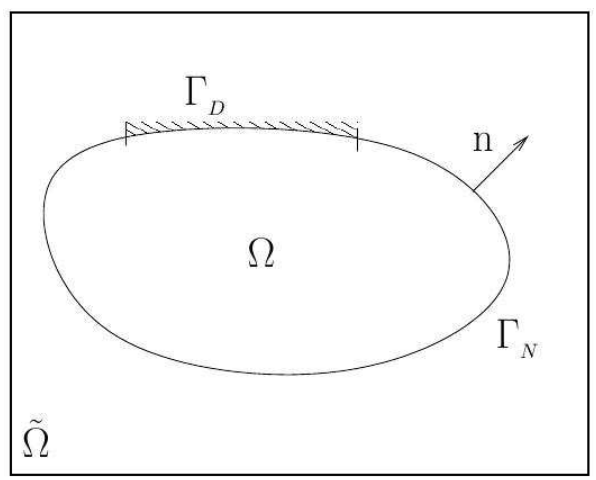

Figure 1: Fictitious and real domains.

For the sake of simplicity, the presentation and the theoretical analysis is made for a twodimensional regular domain $\Omega$, although the method extends naturally to higher dimensions. Let $\widetilde{\Omega} \subset \mathbb{R}^{2}$ be a fictitious domain containing $\Omega$ in its interior (and generally assumed to have a simple shape). We consider that the boundary $\Gamma$ of $\Omega$ is split into two parts $\Gamma_{N}$ and $\Gamma_{D}$ (see Fig. 1). It is assumed that $\Gamma_{D}$ has a nonzero one-dimensional Lebesgue measure. Let us consider the following elliptic problem in $\Omega$ :

$$
\left\{\begin{aligned}
& \text { Find } u: \Omega \mapsto \mathbb{R} \text { such that: } \\
&-\Delta u=f \text { in } \Omega, \\
& u=0 \text { on } \Gamma_{D}, \\
& \partial_{n} u=g \text { on } \Gamma_{N},
\end{aligned}\right.
$$

where $f \in L^{2}(\Omega)$ and $g \in L^{2}\left(\Gamma_{N}\right)$ are given data. The classical weak formulation of this problem 
reads as follows:

$$
\left\{\begin{array}{l}
\text { Find } u \in V \text { and } \lambda \in W \text { such that } \\
a(u, v)+\langle\lambda, v\rangle_{W, X}=l(v) \quad \forall v \in V \\
\langle\mu, u\rangle_{W, X}=0 \quad \forall \mu \in W
\end{array}\right.
$$

where

$$
\begin{gathered}
V=H^{1}(\Omega), X=\left\{w \in L^{2}\left(\Gamma_{D}\right): \exists v \in V, w=v_{\Gamma_{D}}\right\}, \quad W=X^{\prime}, \\
a(u, v)=\int_{\Omega} \nabla u . \nabla v d \Omega, \quad l(v)=\int_{\Omega} f v d \Omega+\int_{\Gamma_{N}} g v d \Gamma,
\end{gathered}
$$

denoting $\langle\mu, v\rangle_{W, X}$ the duality pairing between $W$ and $X$. Classically, Problem (2) is also equivalent to the problem of finding the saddle point of the Lagrangian

$$
\mathcal{L}(v, \mu)=\frac{1}{2} a(v, v)+\langle\mu, v\rangle_{W, X}-l(v),
$$

defined on $V \times X$. The existence and uniqueness of a solution to Problem (2) is obtained by standard techniques.

\section{The fictitious domain method}

The fictitious domain approach requires the introduction of two finite element spaces on the fictitious domain $\widetilde{\Omega}$. Let $\widetilde{V}^{h} \subset H^{1}(\widetilde{\Omega})$ and $\widetilde{W}^{h} \subset L^{2}(\widetilde{\Omega})$ be two finite element spaces. Note that $\widetilde{\Omega}$ may always be chosen as a sufficiently large rectangle $(a, b) \times(c, d)$ such that $\Omega \subset(a, b) \times(c, d)$ which allows $\widetilde{V}^{h}$ and $\widetilde{W}^{h}$ to be defined on the same structured mesh $\mathcal{T}^{h}$ (see Fig. 2). In what follows, we shall suppose that

$$
\widetilde{V}^{h}=\left\{v^{h} \in \mathcal{C}(\widetilde{\widetilde{\Omega}}):\left.v^{h}\right|_{T} \in P(T) \forall T \in \mathcal{T}^{h}\right\},
$$

where $P(T)$ is a finite dimensional space of regular functions satisfying $P(T) \supseteq P_{k}(T)$ for some integer $k \geq 1$.

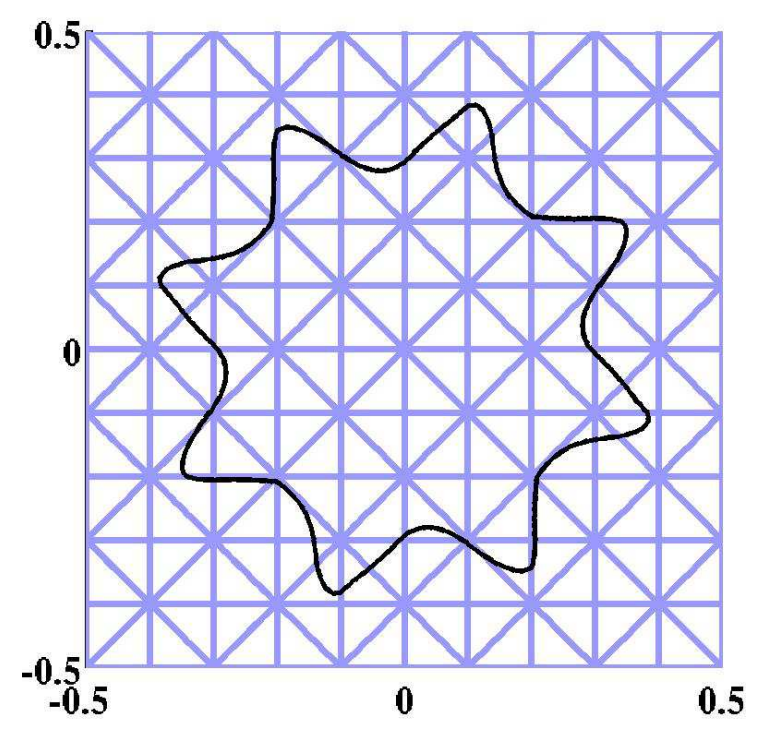

Figure 2: Example of real domain and a structured mesh of the fictitious domain. 
For the approximation on the real domain $\Omega$ we consider the following restriction of $\widetilde{V}^{h}$ and $\widetilde{W}^{h}$ on $\Omega$ and $\Gamma_{D}$, respectively:

$$
V^{h}=\widetilde{V}_{\mid \Omega}^{h}, \text { and } W^{h}=\widetilde{W}_{\left.\right|_{D}}^{h}
$$

which are natural discretizations of $V$ and $W$. An approximation of Problem (2) is then defined as follows:

$$
\left\{\begin{array}{l}
\text { Find } u^{h} \in V^{h} \text { and } \lambda^{h} \in W^{h} \text { such that } \\
a\left(u^{h}, v^{h}\right)+\int_{\Gamma_{D}} \lambda^{h} v^{h} d \Gamma=l\left(v^{h}\right) \quad \forall v^{h} \in V^{h}, \\
\int_{\Gamma_{D}} \mu^{h} u^{h} d \Gamma=0 \quad \forall \mu^{h} \in W^{h} .
\end{array}\right.
$$

We suppose that $\widetilde{W}^{h}$ and $\widetilde{V}^{h}$ are chosen in such a way that the following two conditions are satisfied:

$$
\begin{aligned}
& { }_{1_{\Gamma_{D}} \in W^{h}}, \\
& \bar{\mu}^{h} \in W^{h}: \int_{\Gamma_{D}} \bar{\mu}^{h} v^{h} d \Gamma=0 \quad \forall v^{h} \in V^{h} \Longrightarrow \bar{\mu}^{h}=0 .
\end{aligned}
$$

Without any additional treatment, the following result is proved in [13]:

Proposition 1 Let $\widetilde{V}^{h}$ defined by (4). Suppose that (6) and (7) are satisfied and, in addition

$$
\inf _{\mu^{h} \in W^{h}}\left\|\lambda-\mu^{h}\right\|_{W} \leq h^{\beta}, \quad \beta \geq 1 / 2 .
$$

Then, one has the following error estimate:

$$
\left\|u^{h}-u\right\|_{V} \leq C \sqrt{h}, \quad h \rightarrow 0+
$$

This a priori means that without any treatment, the guaranteed rate of convergence is limited to $O(\sqrt{h})$ which is confirmed is some numerical situations. This reflects a certain kind of numerical locking phenomenon.

\section{$3 \quad$ A local projection stabilized formulation}

In this section we present a new stabilization technique consisting in the addition of a supplementary term involving the local orthogonal projection of the multiplier on a patch decomposition of the mesh.

Let $S^{h}$ be the one-dimensional mesh resulting in the intersection of $\mathcal{T}^{h}$ and $\Gamma_{D}$. The idea is to aggregate the possibly very small elements of $S^{h}$ in order to obtain a set of patches having a minimal and a maximal size (for instance between $3 h$ and $6 h$ ). In practice, this operation can be done rather easily (even for three-dimensional problems). A practical way to obtain such a patch decomposition will be describe in the next section. An example of patch aggregation is presented in Fig. 3.

Let $H$ be the maximum length of these patches and denote by $S^{H}$ the corresponding subdivision of $\Gamma_{D}$. Let

$$
W^{H}=\left\{\mu^{H} \in L^{2}\left(\Gamma_{D}\right):\left.\mu^{H}\right|_{S} \in P_{0}(S), \forall S \in S_{H}\right\},
$$

be the space of piecewise constants on this mesh. This is a classical result, presented in [10], that under a reasonable regularity assumption on $\Gamma_{D}$, an inf-sup condition is satisfied between 
$W^{H}$ and $V^{h}$ for minimal size of $3 h$ for the patches. This implies in particular that an optimal convergence can be reached if the multiplier is taken in $W^{H}$. However, this suppose a relatively coarse approximation of the multiplier. Our approach is to use this result in order to stabilize the approximation obtained with the multiplier defined on the finer discretization $W^{h}$.

Let us first recall the result of Girault and Glowinski in [10]. Under an assumption on $\Gamma_{D}$ to be of class $\mathscr{C}^{1,1}$ and a condition for the patches $S \in S^{H}$ to be approximated by a fixed set of line segments having approximatively the same length (see [10], condition (4.17)) with a length greater or equal to $3 h$ then the following inf-sup (or LBB) condition holds for a constant $\beta^{*}>0$, independent of $h$ and $H$ :

$$
\forall \mu^{H} \in W^{H}, \quad \sup _{v^{h} \in V^{h}} \frac{\int_{\Gamma_{D}} v^{h} \mu^{H} d \Gamma}{\left\|v^{h}\right\|_{V}} \geq \beta^{*}\left\|\mu^{H}\right\|_{-1 / 2, \Gamma_{D}} .
$$

We will assume in the following that the conditions to obtain this inf-sup condition are satisfied.

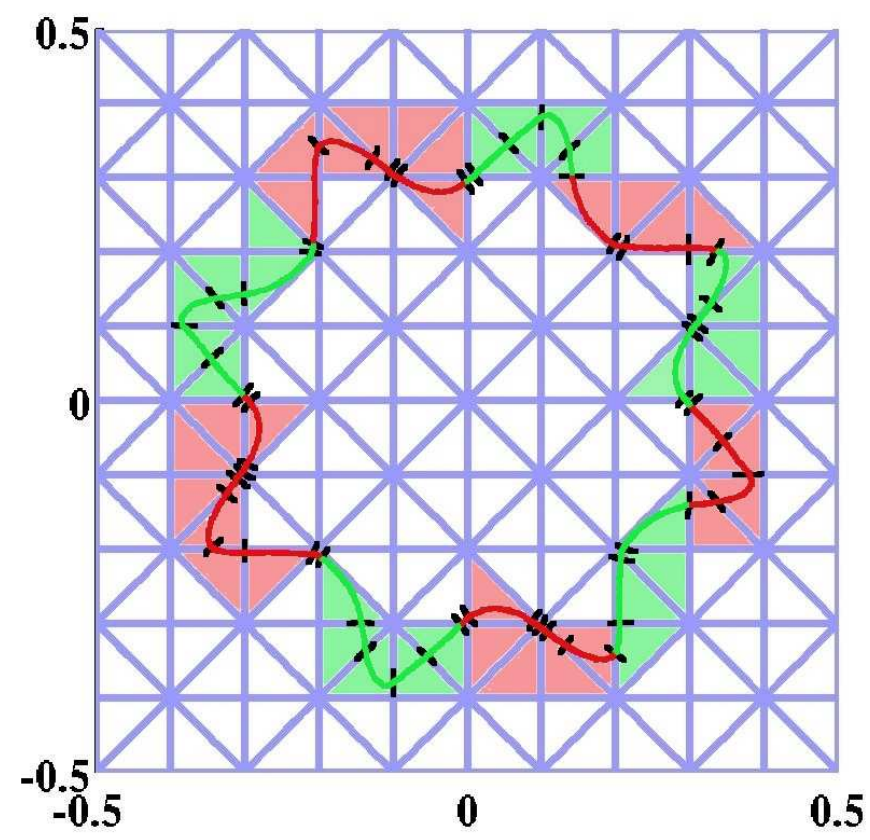

Figure 3: Example of a patch aggregation (in red and green) of size approximatively $2 h$ of the intersection of the boundary of the real domain and the mesh. Note the practically inevitable presence of very small intersections.

Let $P_{W^{H}}$ be the local orthogonal projection operator from $L^{2}\left(\Gamma_{D}\right)$ onto $W^{H}$ which is defined by

$$
\forall \mu \in L^{2}\left(\Gamma_{D}\right),\left.\forall S \in S^{H} \quad P_{W^{H}}(\mu)\right|_{S}=\frac{1}{\operatorname{mes}(S)} \int_{S} \mu d \Gamma .
$$

The stabilized formulation consists in replacing Lagrangian (3) by the following one:

$$
\mathcal{L}_{h}\left(v^{h}, \mu^{h}\right)=\mathcal{L}\left(v^{h}, \mu^{h}\right)-\frac{\gamma}{2} \int_{\Gamma_{D}}\left(\mu_{h}-P_{W^{H}}\left(\mu^{h}\right)\right)^{2} d \Gamma
$$


where, for the sake of simplicity, $\gamma=h \gamma_{0}$ is chosen constant over $\Omega$ (for non-uniform meshes, $\gamma=h_{T} \gamma_{0}$ would be a better choice). The corresponding optimality system reads as follows:

$$
\left\{\begin{array}{l}
\text { Find } u^{h} \in V^{h} \text { and } \lambda^{h} \in W^{h} \text { such that } \\
a\left(u^{h}, v^{h}\right)+\int_{\Gamma_{D}} \lambda^{h} v^{h} d \Gamma=l\left(v^{h}\right) \quad \forall v^{h} \in V^{h}, \\
\int_{\Gamma_{D}} \mu^{h} u^{h} d \Gamma-\gamma \int_{\Gamma_{D}}\left(\lambda^{h}-P_{W^{H}}\left(\lambda^{h}\right)\right)\left(\mu^{h}-P_{W^{H}}\left(\mu^{h}\right)\right) d \Gamma=0 \quad \forall \mu^{h} \in W^{h} .
\end{array}\right.
$$

Lemma 1 Assume that (6) and (8) hold then for any $\gamma_{0}$ there exists a unique solution of the stabilized problem (9).

Proof. Suppose $\left(u_{1}^{h}, \lambda_{1}^{h}\right)$ and $\left(u_{2}^{h}, \lambda_{2}^{h}\right)$ are two solutions to Problem (9). Denoting $\bar{u}^{h}=u_{1}^{h}-u_{2}^{h}$, $\bar{\lambda}^{h}=\lambda_{1}^{h}-\lambda_{2}^{h}$ and $\bar{\lambda}^{H}=P_{W^{H}}\left(\lambda_{1}^{h}\right)-P_{W^{H}}\left(\lambda_{2}^{h}\right)$ then we obtain from Problem (9)

$$
\left\{\begin{array}{l}
a\left(\bar{u}^{h}, \bar{u}^{h}\right)+\int_{\Gamma_{D}} \bar{\lambda}^{h} \bar{u}^{h} d \Gamma=0 \\
\int_{\Gamma_{D}} \bar{\lambda}^{h} \bar{u}^{h} d \Gamma-\gamma \int_{\Gamma_{D}}\left(\bar{\lambda}^{h}-\bar{\lambda}^{H}\right)^{2} d \Gamma=0 \quad \forall \mu^{h} \in W^{h} .
\end{array}\right.
$$

Consequently,

$$
a\left(\bar{u}^{h}, \bar{u}^{h}\right)+\gamma \int_{\Gamma_{D}}\left(\bar{\lambda}^{h}-\bar{\lambda}^{H}\right)^{2} d \Gamma=0,
$$

which implies $\bar{u}^{h}=0$ and $\bar{\lambda}^{h}=\bar{\lambda}^{H}$ (i.e. $\bar{\lambda}^{h} \in W^{H}$ ). Moreover, from (8) there exist $v^{h} \in V^{h}$ such that

$$
\int_{\Gamma_{D}} \bar{\lambda}^{H} v^{h} \geq \beta^{*}\left\|\bar{\lambda}^{H}\right\|_{-1 / 2, \Gamma_{D}}\left\|v^{h}\right\|_{V}
$$

and thus

$$
\beta^{*}\left\|\bar{\lambda}^{H}\right\|_{-1 / 2, \Gamma_{D}} \leq \frac{1}{\left\|v^{h}\right\|_{V}} \int_{\Gamma_{D}} \bar{\lambda}^{H} v^{h} d \Gamma=\frac{1}{\left\|v^{h}\right\|_{V}} \int_{\Gamma_{D}} \bar{\lambda}^{h} v^{h} d \Gamma=\frac{1}{\left\|v^{h}\right\|_{V}} a\left(\bar{u}^{h}, v^{h}\right)=0 .
$$

This implies the uniqueness of the solution and, since the dimension of the linear system (9) is finite, then existence as well.

\subsection{Convergence analysis}

In this section, we establish an optimal a priori error estimate for the following standard finite element spaces:

$$
\begin{gathered}
\widetilde{V}^{h}=\left\{v^{h} \in \mathcal{C}(\widetilde{\Omega}):\left.v^{h}\right|_{T} \in P(T) \forall T \in \mathcal{T}^{h}\right\}, \\
\widetilde{W}^{h}=\left\{\mu^{h} \in L^{2}(\widetilde{\Omega}):\left.\mu^{h}\right|_{T} \in P^{\prime}(T) \forall T \in \mathcal{T}^{h}\right\},
\end{gathered}
$$

where $P(T)$ (rep. $P^{\prime}(T)$ ) is a finite dimensional space of regular functions satisfying $P(T) \supseteq P_{k}(T)$ (resp. $P(T) \supseteq P_{k^{\prime}}(T)$ ) for an integer $k \geq 1$ (resp. $k^{\prime} \geq 0$ ).

Theorem 1 Let $\widetilde{V}^{h}$ and $\widetilde{W}^{h}$ be defined by (13) and (14), respectively. Let $(u, \lambda)$ be the solution of the continuous problem (2) such that $u \in H^{2}(\Omega)$ and $\lambda \in H^{1 / 2}\left(\Gamma_{D}\right)$. Assume that (8) and (6) are satisfied and assume also the existence of a constant $\eta>1$ with $H \leq \eta h$. Then the following estimate holds for $C>0$ a constant independent of $h$ :

$$
\left|\left\|\left(u-u^{h}, \lambda-\lambda^{h}\right)\right\|\right| \leq C h\left(\|u\|_{2, \Omega}+\|\lambda\|_{1 / 2, \Gamma_{D}}\right),
$$

where $|\|(u, \lambda)\||^{2}=\|u\|_{V}^{2}+\|\lambda\|_{-1 / 2, \Gamma_{D}}^{2}$ and $\left(u^{h}, \lambda^{h}\right)$ is the solution to Problem (9). 
Proof. For all $v^{h} \in V^{h}$ and $\mu^{H} \in W^{H}$ we have:

$$
\begin{aligned}
\alpha\left\|u^{h}-u\right\|_{V}^{2} \leq & a\left(u^{h}-u, u^{h}-u\right)=a\left(u^{h}-u, v^{h}-u\right)+a\left(u^{h}-u, u^{h}-v^{h}\right), \\
\leq & M\left\|u^{h}-u\right\|_{V}\left\|v^{h}-u\right\|_{V}-\int_{\Gamma_{D}}\left(\lambda^{h}-\lambda\right)\left(u^{h}-v^{h}\right) d \Gamma \\
= & M\left\|u^{h}-u\right\|_{V}\left\|v^{h}-u\right\|_{V}-\int_{\Gamma_{D}} \lambda^{h} u^{h} d \Gamma+\int_{\Gamma_{D}} \lambda u^{h} d \Gamma+\int_{\Gamma_{D}}\left(\lambda^{h}-\lambda\right)\left(v^{h}-u\right) d \Gamma, \\
= & M\left\|u^{h}-u\right\|_{V}\left\|v^{h}-u\right\|_{V}-\gamma\left\|\lambda^{h}-\lambda^{H}\right\|_{0, \Gamma_{D}}^{2}+\int_{\Gamma_{D}}\left(\lambda-\mu^{H}\right)\left(u^{h}-u\right) d \Gamma \\
& \quad+\int_{\Gamma_{D}}\left(\lambda^{h}-\lambda\right)\left(v^{h}-u\right) d \Gamma,
\end{aligned}
$$

because in particular $\int_{\Gamma_{D}}\left(\lambda^{h}-\lambda\right) u d \Gamma=0$. Then, still for all $v^{h} \in V^{h}$ and $\mu^{H} \in W^{H}$ we deduce

$$
\begin{aligned}
\alpha\left\|u^{h}-u\right\|_{V}^{2}+ & \gamma\left\|\lambda^{h}-\lambda^{H}\right\|_{-1 / 2, \Gamma_{D}}^{2} \leq M\left\|u^{h}-u\right\|_{V}\left\|v^{h}-u\right\|_{V} \\
& +\left\|\lambda-\mu^{H}\right\|_{-1 / 2, \Gamma_{D}}\left\|u^{h}-u\right\|_{V}+\left\|\lambda^{h}-\lambda\right\|_{-1 / 2, \Gamma_{D}}\left\|u-v^{h}\right\|_{V} .
\end{aligned}
$$

Beside,

$$
\int_{\Gamma_{D}}\left(\lambda-\lambda^{h}\right) v^{h} d \Gamma=a\left(u^{h}-u, v^{h}\right) \quad \forall v^{h} \in V^{h}
$$

and therefore one obtains

$$
\int_{\Gamma_{D}}\left(\bar{\mu}^{h}-\lambda^{h}\right) v^{h} d \Gamma=a\left(u^{h}-u, v^{h}\right)+\int_{\Gamma_{D}}\left(\bar{\mu}^{h}-\lambda\right) v^{h} d \Gamma \quad \forall v^{h} \in V^{h} ; \forall \bar{\mu}^{h} \in W^{h} .
$$

Now, for $\mu^{H}=\lambda^{H}-\bar{\mu}^{H} \in W^{H}$ with $\bar{\mu}^{H}=P_{W^{H}}\left(\bar{\mu}^{h}\right)$ the inf-sup condition (8) ensure the existence of $v^{h} \in V^{h}$ such that together with (17) we get

$$
\begin{aligned}
\beta^{*}\left\|\lambda^{H}-\bar{\mu}^{H}\right\|_{-1 / 2, \Gamma_{D}} & \leq \frac{1}{\left\|v^{h}\right\|_{V}} \int_{\Gamma_{D}}\left(\bar{\mu}^{H}-\lambda^{H}\right) v^{h} d \Gamma \\
& \leq \frac{1}{\left\|v^{h}\right\|_{V}} \int_{\Gamma_{D}}\left(\bar{\mu}^{h}-\lambda^{h}\right) v^{h} d \Gamma+\frac{1}{\left\|v^{h}\right\|_{V}} \int_{\Gamma_{D}}\left(\bar{\mu}^{H}-\lambda^{H}-\left(\bar{\mu}^{h}-\lambda^{h}\right)\right) v^{h} d \Gamma, \\
& \leq M\left\|u^{h}-u\right\|_{V}+\left\|\bar{\mu}^{h}-\lambda\right\|_{-1 / 2, \Gamma_{D}}+\left\|\bar{\mu}^{H}-\lambda^{H}-\left(\bar{\mu}^{h}-\lambda^{h}\right)\right\|_{-1 / 2, \Gamma_{D}} .
\end{aligned}
$$

As a consequence, one has

$$
\begin{aligned}
\beta^{*}\left\|\lambda^{H}-\lambda\right\|_{-1 / 2, \Gamma_{D} \leq} \leq & \beta^{*}\left\|\lambda-\bar{\mu}^{H}\right\|_{-1 / 2, \Gamma_{D}}+M\left\|u^{h}-u\right\|_{V}+\left\|\bar{\mu}^{h}-\lambda\right\|_{-1 / 2, \Gamma_{D}} \\
& +\left\|\bar{\mu}^{H}-\bar{\mu}^{h}\right\|_{-1 / 2, \Gamma_{D}}+\left\|\lambda^{H}-\lambda^{h}\right\|_{-1 / 2, \Gamma_{D}},
\end{aligned}
$$

and

$$
\begin{aligned}
\beta^{* 2}\left\|\lambda^{H}-\lambda\right\|_{-1 / 2, \Gamma_{D}}^{2} \leq & 8 M^{2}\left\|u-u^{h}\right\|_{V}^{2}+8 \beta^{* 2}\left\|\lambda-\bar{\mu}^{H}\right\|_{-1 / 2, \Gamma_{D}}^{2}+8\left\|\lambda-\bar{\mu}^{h}\right\|_{-1 / 2, \Gamma_{D}}^{2} \\
& +8\left\|\bar{\mu}^{H}-\bar{\mu}^{h}\right\|_{-1 / 2, \Gamma_{D}}^{2}+8\left\|\lambda^{H}-\lambda^{h}\right\|_{-1 / 2, \Gamma_{D}}^{2} \quad \forall \bar{\mu}^{h} \in W^{h} .
\end{aligned}
$$


By combining inequalities (16) and (18) one obtains for all $\bar{\mu}^{h} \in W^{h}, \mu^{H} \in W^{H}$ and $v^{h} \in V^{h}$

$$
\begin{aligned}
& \left(\alpha-8 M^{2} \delta\right)\left\|u-u^{h}\right\|_{V}^{2}+\delta \beta^{* 2}\left\|\lambda-\lambda^{H}\right\|_{-1 / 2, \Gamma_{D}}^{2}+(\gamma-8 \delta)\left\|\lambda^{h}-\lambda^{H}\right\|_{-1 / 2, \Gamma_{D}}^{2} \\
\leq & M\left\|u^{h}-u\right\|_{V}\left\|v^{h}-u\right\|_{V}+\left\|\lambda-\mu^{H}\right\|_{-1 / 2, \Gamma_{D}}\left\|u^{h}-u\right\|_{V}+\left\|\lambda-\lambda^{h}\right\|_{-1 / 2, \Gamma_{D}}\left\|u-v^{h}\right\|_{V} \\
& +8 \delta \beta^{* 2}\left\|\lambda-\bar{\mu}^{H}\right\|_{-1 / 2, \Gamma_{D}}^{2}+8 \delta\left\|\lambda-\bar{\mu}^{h}\right\|_{-1 / 2, \Gamma_{D}}^{2}+8 \delta\left\|\bar{\mu}^{h}-\bar{\mu}^{H}\right\|_{-1 / 2, \Gamma_{D}}^{2}, \\
\leq & \frac{\delta}{2} M^{2}\left\|u-u^{h}\right\|_{V}^{2}+\frac{1}{2 \delta}\left\|u-v^{h}\right\|_{V}^{2}+\frac{\delta}{2}\left\|u-u^{h}\right\|_{V}^{2}+\frac{1}{2 \delta}\left\|\lambda-\mu^{H}\right\|_{-1 / 2, \Gamma_{D}}^{2}+\frac{\xi}{2}\left\|\lambda-\lambda^{h}\right\|_{-1 / 2, \Gamma_{D}}^{2} \\
& +\frac{1}{2 \xi}\left\|u-v^{h}\right\|_{V}^{2}+8 \delta \beta^{* 2}\left\|\lambda-\bar{\mu}^{H}\right\|_{-1 / 2, \Gamma_{D}}^{2}+8 \delta\left\|\lambda-\bar{\mu}^{h}\right\|_{-1 / 2, \Gamma_{D}}^{2}+8 \delta\left\|\bar{\mu}^{h}-\bar{\mu}^{H}\right\|_{-1 / 2, \Gamma_{D}}^{2} .
\end{aligned}
$$

Let $\delta$ and $\xi$ be such that $\delta<\frac{2 \alpha}{17 M^{2}+1}$ and $\xi<\min \left(\frac{2 \alpha \beta^{* 2}}{17 M^{2}+1} ; \frac{\gamma \beta^{* 2}}{\beta^{* 2}+8}\right)$, then, still for all $\bar{\mu}^{h} \in W^{h}, \mu^{H} \in W^{H}$ and $v^{h} \in V^{h}$, one deduces

$$
\begin{aligned}
& \left(\alpha-\delta \frac{17 M^{2}+1}{2}\right)\left\|u-u^{h}\right\|_{V}^{2}+(\gamma-8 \delta-\xi)\left\|\lambda^{h}-\lambda^{H}\right\|_{-1 / 2, \Gamma_{D}}^{2}+\left(\delta \beta^{* 2}-\xi\right)\left\|\lambda-\lambda^{H}\right\|_{-1 / 2, \Gamma_{D}}^{2} \\
\leq & \left(\frac{1}{2 \delta}+\frac{1}{2 \xi}\right)\left\|u-v^{h}\right\|_{V}^{2}+\frac{1}{2 \delta}\left\|\lambda-\mu^{H}\right\|_{-1 / 2, \Gamma_{D}}^{2}+8 \delta \beta^{* 2}\left\|\lambda-\bar{\mu}^{H}\right\|_{-1 / 2, \Gamma_{D}}^{2}+8 \delta\left\|\lambda-\bar{\mu}^{h}\right\|_{-1 / 2, \Gamma_{D}}^{2} \\
& +8 \delta\left\|\bar{\mu}^{h}-\bar{\mu}^{H}\right\|_{-1 / 2, \Gamma_{D}}^{2}, \quad \forall \bar{\mu}^{h} \in W^{h} .
\end{aligned}
$$

Denoting by $\Pi^{h}$ (resp. $\left.P_{W^{h}}\right)$ the Lagrange interpolation operator (resp. the $L^{2}\left(\Gamma_{D}\right)$-projection) in $V^{h}$ (resp. in $W^{h}$ ), we have the following standard finite element estimates:

$$
\begin{gathered}
\left\|u-\Pi^{h} u\right\|_{V} \leq C h\|u\|_{2, \Omega}, \\
\left\|\lambda-P_{w^{h}}(\lambda)\right\|_{-1 / 2, \Gamma_{D}} \leq C h\|\lambda\|_{1 / 2, \Gamma_{D}}, \\
\left\|\lambda-P_{w^{H}}(\lambda)\right\|_{-1 / 2, \Gamma_{D}} \leq C H\|\lambda\|_{1 / 2, \Gamma_{D}} .
\end{gathered}
$$

Finally, the theorem is established by taking $v^{h}=\Pi^{h} u, \bar{\mu}^{h}=P_{w^{h}}(\lambda)$ and $\mu^{H}=P_{w^{H}}(\lambda)$.

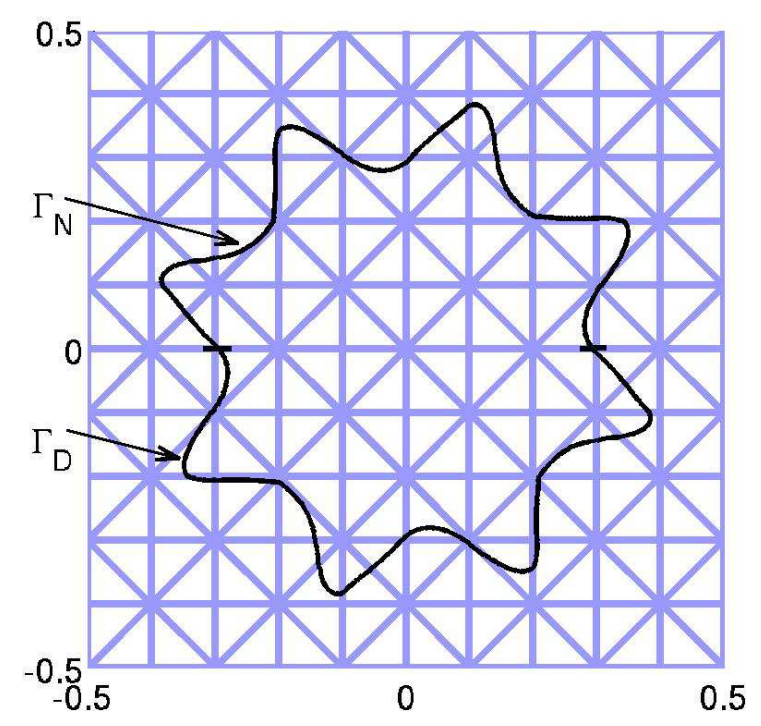

Figure 4: Example of a two-dimensionnal triangular structured mesh used for the numerical test and partition of the boundary for Neumann and Dirichlet conditions. 


\section{$4 \quad$ Numerical tests}

In this section, we present $2 \mathrm{D}$ and $3 \mathrm{D}$-numerical tests for a fictitious domain being $\widetilde{\Omega}=]-$ $1 / 2,1 / 2\left[{ }^{d}\right.$ for $d=2$ and $d=3$, respectively. The bidimensionnal exact solution is chosen to be $u(x)=5 *\left(R^{4}-r^{4}\left(2.5+1.5 * \sin \left(8 * \theta+\frac{2 \pi}{9}\right)\right)\right)$ where $r=\sqrt{x_{1}^{2}+x_{2}^{2}}, R=0.47$ and the three-dimensional one $u(x)=5 *\left(R^{3}-\rho^{3}\right)$ with $\rho=\sqrt{x_{1}^{2}+x_{2}^{2}+x_{3}^{3}}$. In both cases, the real domain is $\Omega=\left\{x \in \mathbb{R}^{d}: u(x)<0\right\}$ and the Dirichlet and Neumann boundary conditions are defined on $\Gamma_{D}=\Gamma \cap\left\{x \in \mathbb{R}^{d}: x_{d}<0\right\}$ and $\Gamma_{N}=\Gamma \cap\left\{x \in \mathbb{R}^{d}: x_{d}>0\right\}$, respectively. The two-dimensional domain is represented in Fig. 4 with an example of a triangular structured mesh. The exact solutions are shown in Fig. 5.

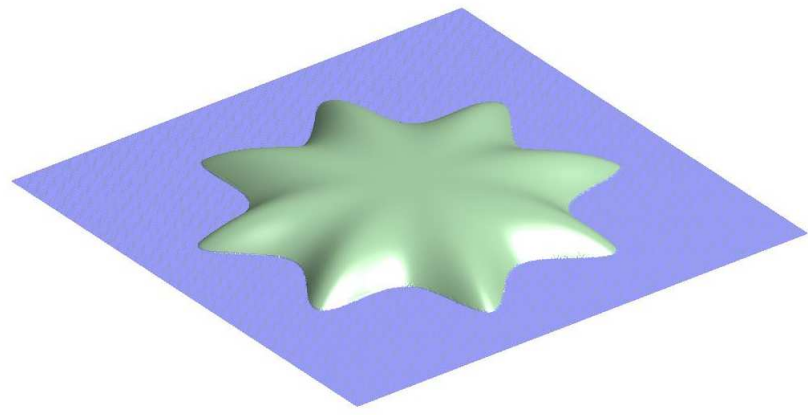

(a) Two-dimensional exact solution

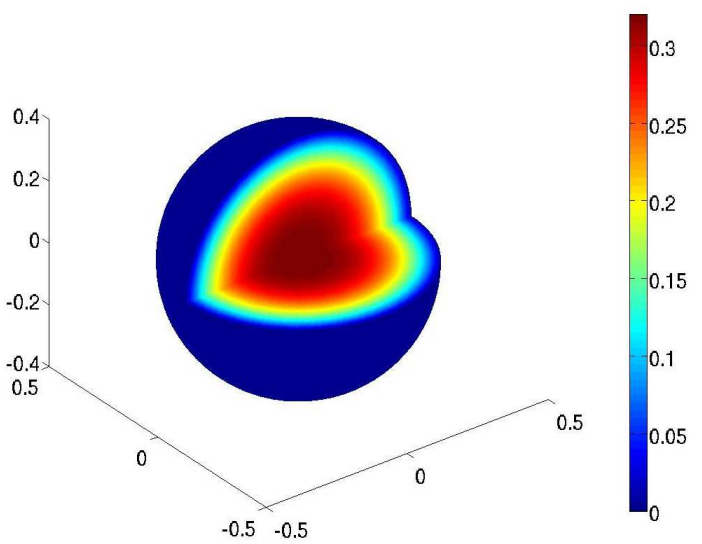

(b) Three-dimensional exact solution

Figure 5: Exact solutions

The numerical tests are performed with GETFEM++, the $\mathrm{C}++$ finite-element library developed by our team (see [22]).

\subsection{Numerical solving}

The algebraic formulation of Problem (9) reads

$$
\left\{\begin{array}{l}
\text { Find } U \in \mathbb{R}^{N_{u}} \text { and } L \in \mathbb{R}^{N_{\lambda}} \text { such that } \\
K U+B^{T} L=F \\
B U-M_{\gamma} L=0
\end{array}\right.
$$

where $U$ is the vector of degrees of freedom for $u^{h}, L$ the one for the multiplier $\lambda^{H}, N_{u}$ and $N_{\lambda}$ the dimensions of $V^{h}$ and $W^{h}$, respectively, $K$ is the stiffness matrix coming from the term $a\left(u^{h}, v^{h}\right)$, $F$ is the right-hand side and the term $\ell\left(v^{h}\right)$, and $B$ and $M_{\gamma}$ are the matrices corresponding to the terms $\int_{\Gamma_{D}} \lambda^{H} v^{h} d \Gamma$ and $\gamma \int_{\Gamma_{D}}\left(\lambda^{h}-P_{W^{H}}\left(\lambda^{h}\right)\right)\left(\mu^{h}-P_{W^{H}}\left(\mu^{h}\right)\right) d \Gamma$, respectively.

Before presenting the numerical experiments, we shall describe in details two important aspects of the implementation of the method. Namely, the extraction of a basis for $W^{h}$ and the repartition of the element having an intersection with $\Gamma_{D}$ into patches.

The extraction of a basis of $W^{h}$ could be non-trivial in some cases, except when a piecewise constants $\left(P_{0}\right)$ finite element method is used to approximate the multiplier or in some other cases when $\Gamma_{D}$ is curved. Indeed, if one selects all the shape functions of $\widetilde{W}^{h}$ whose support intersects 
$\Gamma_{D}$, some of them can be linearly dependent, especially when $\Gamma_{D}$ is a straight line. In order to eliminate linearly dependent shape functions, the choice here is to consider the mass matrix $\int_{\Gamma_{D}} \psi_{i} \psi_{j} d \Gamma$ where the $\psi_{i}$ are the finite-element shape functions of $\widetilde{W}^{h}$. A block-wise GramSchmidt algorithm is used to eliminate local dependencies and then the potential remaining kernel of the mass matrix is detected by a Lanczos algorithm. In the presented numerical tests, since curved boundaries are considered the kernel of the mass matrix is either reduced to 0 or very small. In [1] some numerical experiments are presented for a straight line in $2 \mathrm{D}$ using the same technique. The selection of a basis of $W^{h}$ using this technique took far less computational time than the assembly of the stiffness matrix.

The decomposition into patches is made using a graph partitioner algorithm. In the presented numerical tests we use the free software METIS [14]. The nodes of the graph consists in the elements having an intersection with $\Gamma_{D}$ and the edges connect adjacent elements. Additionally, a load corresponding to the size of the intersection is considered on each elements. The partition is a very fast operation.

\subsection{Comparison with the Barbosa-Hughes stabilization technique}

In our numerical test, we compare the new stabilization technique to the one studied in [13] in the same framework which use the technique introduced by Barbosa and Hughes in [4, 5]. For the self consistency of the paper, we recall briefly the principle of the symmetric version of the Barbosa-Hughes stabilization technique applied to Problem (5) has it is presented in [13].

This technique is based on the addition of a supplementary term involving an approximation of the normal derivative on $\Gamma_{D}$. Let us suppose that we have at our disposal an operator

$$
R^{h}: V^{h} \longrightarrow L^{2}\left(\Gamma_{D}\right),
$$

which approximates the normal derivative on $\Gamma_{D}$ (i.e. for $v^{h} \in V^{h}$ converging to a sufficiently smooth function $v, R^{h}\left(v^{h}\right)$ tends to $\partial_{n} v$ in an appropriate sense). Several choices of $R^{h}$ are proposed in [13]. To obtain the stabilized problem, the Lagrangian (3) is replaced by the following one

$$
\mathcal{L}_{h}\left(v^{h}, \mu^{h}\right)=\mathcal{L}\left(v^{h}, \mu^{h}\right)-\frac{\gamma}{2} \int_{\Gamma_{D}}\left(\mu^{h}+R^{h}\left(v^{h}\right)\right)^{2} d \Gamma, \quad v^{h} \in V^{h}, \mu^{h} \in W^{h},
$$

where for the sake of simplicity $\gamma:=h \gamma_{0}$ is still chosen to be a positive constant over $\Omega$. The corresponding discrete problem reads as follows:

$$
\left\{\begin{array}{l}
\text { Find } u^{h} \in V^{h} \text { and } \lambda^{h} \in W^{h} \text { such that } \\
a\left(u^{h}, v^{h}\right)+\int_{\Gamma_{D}} \lambda^{h} v^{h} d \Gamma-\gamma \int_{\Gamma_{D}}\left(\lambda^{h}+R^{h}\left(u^{h}\right)\right) R^{h}\left(v^{h}\right) d \Gamma=l\left(v^{h}\right) \quad \forall v^{h} \in V^{h}, \\
\int_{\Gamma_{D}} \mu^{h} u^{h} d \Gamma-\gamma \int_{\Gamma_{D}}\left(\lambda^{h}+R^{h}\left(u^{h}\right)\right) \mu^{h} d \Gamma=0 \quad \forall \mu^{h} \in W^{h} .
\end{array}\right.
$$

More details and a convergence analysis can be found in [13]. Note that this is also a consistent modification of the Lagrangian and that a close relationship between Barbosa-Hughes stabilization technique and Nitsche's one [19] has been explicited in [25].

\subsection{Two-dimensional numerical tests}

A comparison is done between the non-stabilized problem (5), the local projection stabilized problem (9) and the Barbosa-Hughes stabilized one (20) in the two-dimensional case. Additionally, we test different pairs of elements for the main unknown $u$ and the multiplier. Namely, we test 
the following methods: $P_{2} / P_{1}, P_{1} / P_{1}, P_{1} / P_{0} . P_{1} / P_{2}, Q_{1} / Q_{0}$ and $Q_{1} / Q_{0}$. The notation $P_{i} / P_{j}$ (resp. $Q_{i} / Q_{j}$ ) means that solution $u$ is approximated with a $P_{i}$ finite-element method (resp. a $Q_{i}$ finite-element method) and the multiplier with a continuous $P_{j}$ finite-element method (resp. continuous $Q_{j}$ finite-element method).

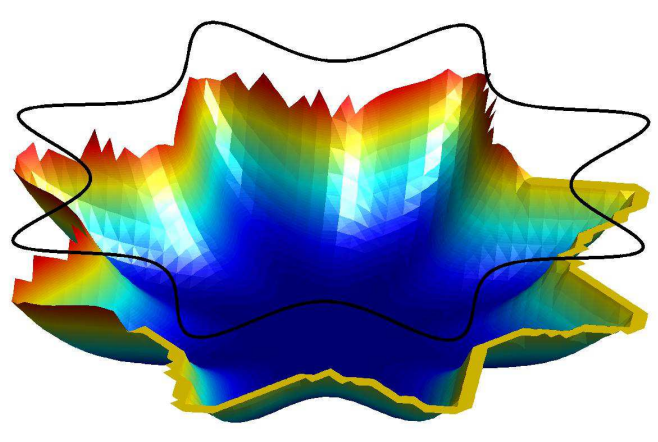

(a) Solution on $\Omega$ with no stabilization for a $P_{1} / P_{2}$ method.

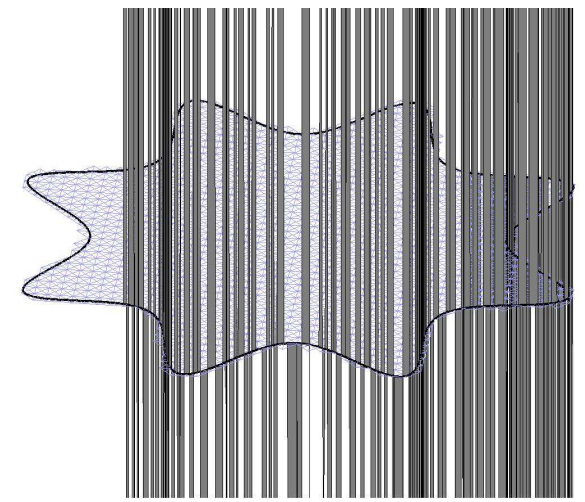

(b) Multiplier on $\Gamma_{D}$ with no stabilization for a $P_{1} / P_{2}$ method.

Figure 6: Non-stabilized case with a $P_{1} / P_{2}$ method.

Without stabilization. A solution is plotted in Fig. 6 for a $P_{1} / P_{2}$ method. Of course, for such a pair of elements, a uniform discrete inf-sup cannot be satisfied since the multiplier is discretized with a reacher method than the main unknown. Has a consequence, a local locking phenomenon (Fig. 6(a)) on the Dirichlet boundary (a flat solution) holds together with a very noisy multiplier (Fig. 6(b)). This denotes the presence of spurious modes. Some similar results can be observed with $P_{1} / P_{1}$ and $P_{1} / P_{0}$ methods.

The convergence curves in the non-stabilized case are given in Fig. 7(a) for the error in the $L^{2}(\Omega)$-norm on $u$, in Fig. 7(b) for the error in the $H^{1}(\Omega)$-norm on $u$ and in Fig. $7(\mathrm{c})$ for the error in the $L^{2}\left(\Gamma_{D}\right)$-norm on the multiplier. One notes that the convergence rate for the $P_{1} / P_{2}$, $P_{1} / P_{1}$ and $P_{1} / P_{0}$ methods in $H^{1}(\Omega)$-norm are close to 0.5 which is in good accordance with the general result of Proposition 1. In this cases, there is no convergence of the multiplier (still due to the presence of some spurious modes). Conversely, for $P_{2} / P_{1}, Q_{2} / Q_{1}$ and $Q_{1} / Q_{0}$ methods, one observes a nearly optimal convergence rate. This do not imply that a mesh independent inf-sup condition is systematically satisfied in these cases. In [13], some numerical experiments shows that the solution can be deteriorated in the vicinity of very small intersections between the mesh and $\Gamma_{D}$ (especially for the multiplier).

Barbosa-Hughes stabilization. Fig. 8 shows that Barbosa-Hughes stabilization technique eliminates the locking phenomenon (Fig. 8(a)) and the spurious modes on the multiplier (Fig. 8(b)). The convergence curves in the Barbosa-Hughes stabilized case are given in Fig. 9(a) for the error in the $L^{2}(\Omega)$-norm on $u$, in Fig. 9(b) for the error in the $H^{1}(\Omega)$-norm on $u$ and in Fig. 9(c) for the error in the $L^{2}\left(\Gamma_{D}\right)$-norm on the multiplier. The rate of convergence for the error in $L^{2}(\Omega)$-norm (resp. $H^{1}(\Omega)$-norm) on $u$ with Barbosa-Hughes stabilization are optimal of order 3 (resp. of order close to 2) for both $P_{2} / P_{1}$ and $Q_{2} / Q_{1}$ and of order 2 (resp. order 1) for the remaining pairs of elements. Fig. 9(c) shows that the approximation of the multiplier is considerably improved. Concerning the error in $L^{2}\left(\Gamma_{D}\right)$-norm for the multiplier the rate of convergence is also close to optimality for all pairs of elements.

We refer to [1] for the study of the influence of the stabilization parameter. A rather small influence is noted on the error in $L^{2}(\Omega)$ and $H^{1}(\Omega)$-norms on $u$. Concerning the error in $L^{2}\left(\Gamma_{D}\right)$ norm of the multiplier, the value of the stabilization parameter can be divided into two zones. 
A coercive zone where the error decreases when the stabilization parameter $\gamma_{0}$ increases and a non-coercive zone for large values of the stabilization parameter where the error evolves randomly according to the stabilization parameter.

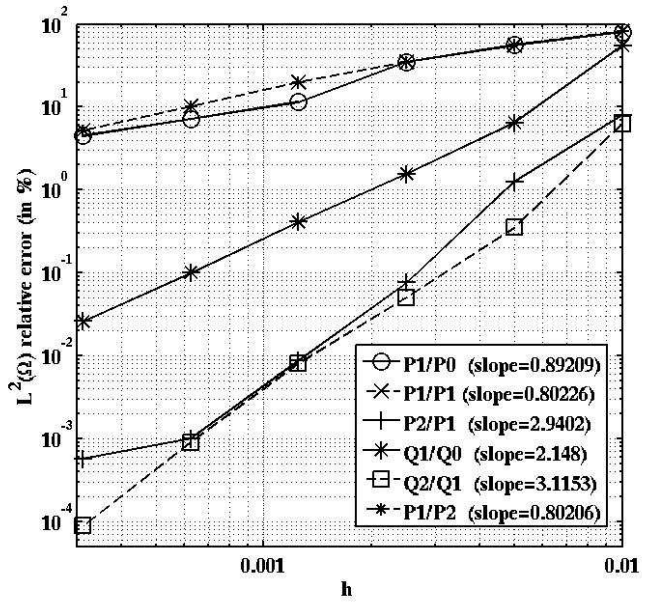

(a) Convergence of $\left\|u-u^{h}\right\|_{0, \Omega}$

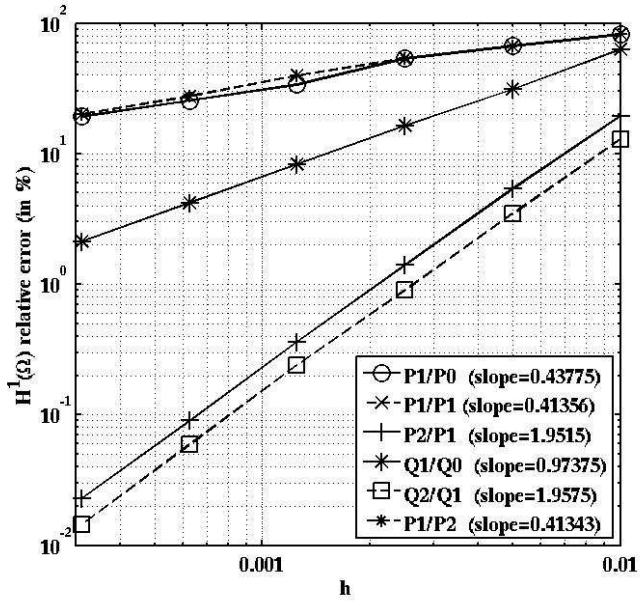

(b) Convergence of $\left\|u-u^{h}\right\|_{1, \Omega}$

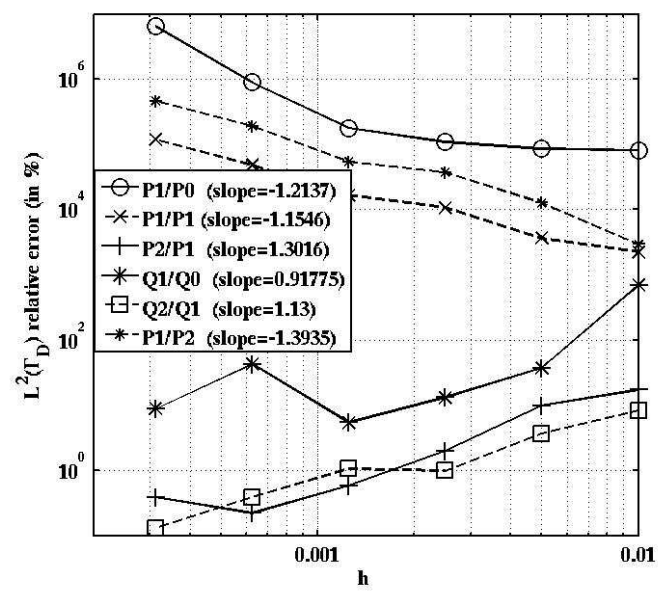

(c) Convergence of $\left\|\lambda^{h}-\lambda\right\|_{0, \Gamma_{D}}$

Figure 7: Convergence curves in the non-stabilized case. 


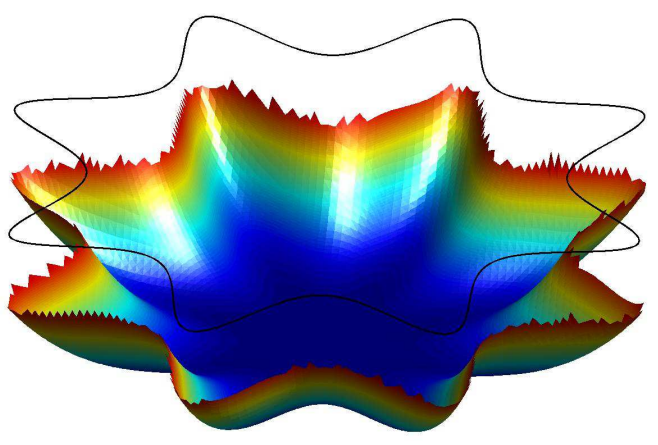

(a) Solution on $\Omega$ with Barbosa-Hughes stabilization for $P_{1} / P_{1}$ method.

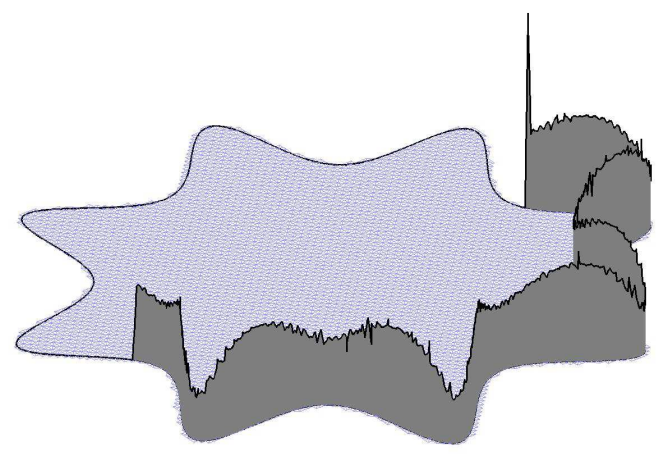

(b) Multiplier on $\Gamma_{D}$ with Barbosa-Hughes stabilization for $P_{1} / P_{1}$ method.

Figure 8: Barbosa-Hughes stabilized case with a $P_{1} / P_{1}$ method.

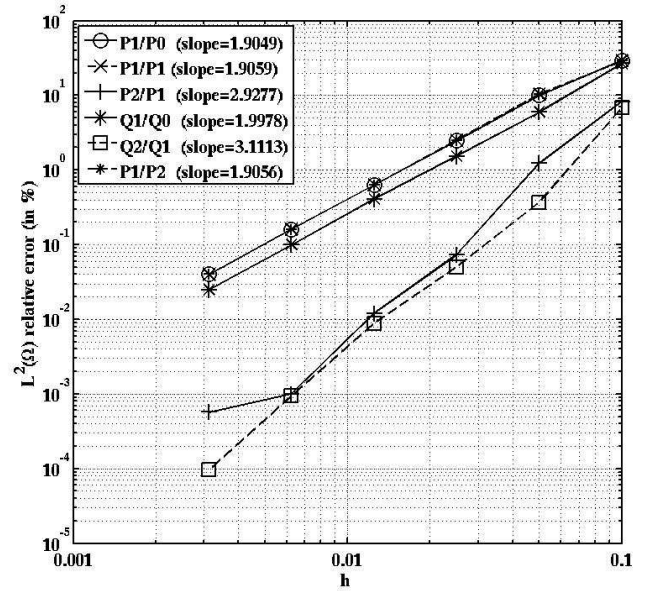

(a) Convergence of $\left\|u-u^{h}\right\|_{0, \Omega}$

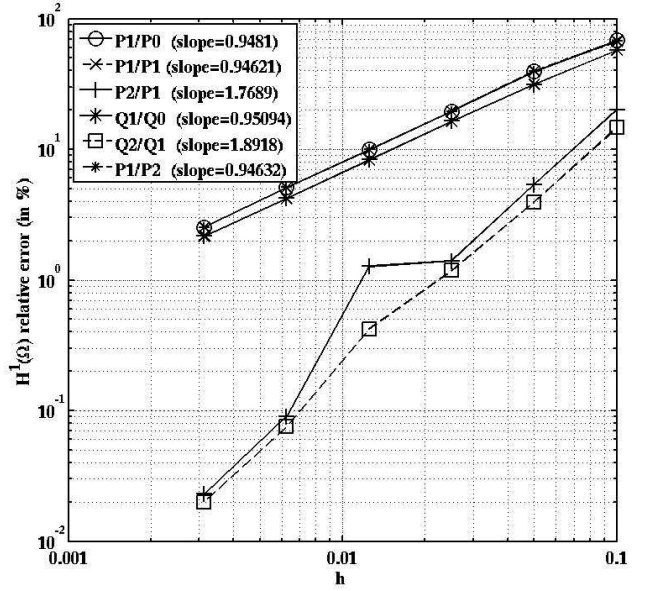

(b) Convergence of $\left\|u-u^{h}\right\|_{1, \Omega}$

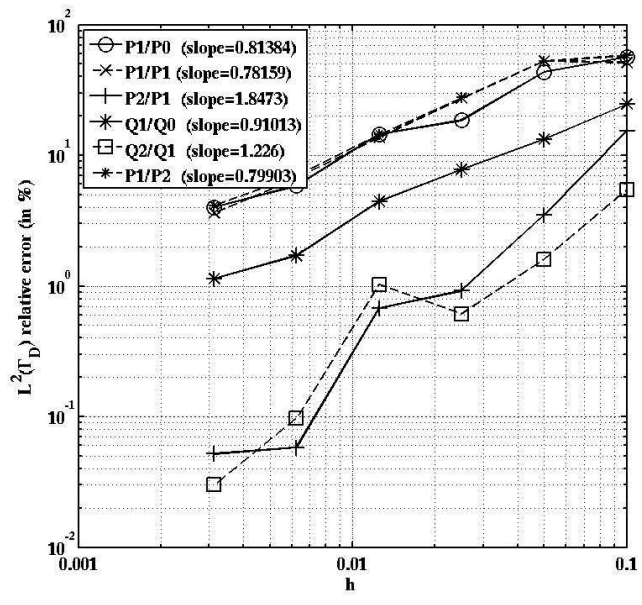

(c) Convergence of $\left\|\lambda^{h}-\lambda\right\|_{0, \Gamma_{D}}$

Figure 9: Convergence curves in the Barbosa-Hughes stabilized case. 


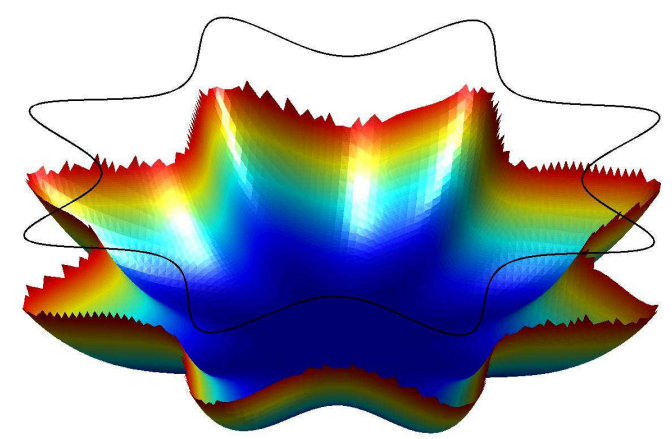

(a) Solution on $\Omega$ with local projection stabilization for $P_{1} / P_{1}$ method.

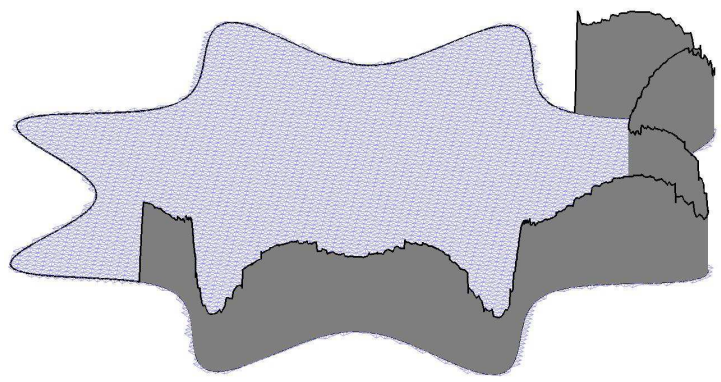

(b) Multiplier on $\Gamma_{D}$ with local projection stabilization for $P_{1} / P_{1}$ method.

Figure 10: Local projection stabilized case with a $P_{1} / P_{1}$ method.

Local projection stabilization. Similarly to the Barbosa-Hughes stabilization, the local projection stabilization gives some optimal rates of convergence for all pairs of elements and eliminates the locking phenomena (Fig. 10(a)) and the spurious modes on the multiplier (Fig. $8(\mathrm{~b})$ ). The convergence curves are shown in Fig. 11(a) for the error in the $L^{2}(\Omega)$-norm on $u$, in Fig. 11(b) for the error in the $H^{1}(\Omega)$-norm on $u$ and in Fig. 11(c) for the error in the $L^{2}\left(\Gamma_{D}\right)$-norm on the multiplier. The rate of convergence for the $P_{1} / P_{2}, P_{1} / P_{1}, P_{1} / P_{0}$ and $Q_{1} / Q_{0}$ methods are in good accordance with the theoretical result of Theorem 1. For $P_{2} / P_{1}$ and $Q_{2} / Q_{1}$ methods, the rates are close to optimality. For these methods, if one tries to extend the result of Theorem 1 to a $H^{3}(\Omega)$ regular exact solution, one find that the rate of convergence of the error estimate depends on the error interpolation of the local orthogonal projection which limits the rate of convergence to $3 / 2$ for the $H^{1}(\Omega)$-norm and 1 for the $L^{2}\left(\Gamma_{D}\right)$-norm on the multiplier. This limitation is observed on Fig. 11(c) on the multiplier of the $Q_{2} / Q_{1}$ method, but not for the $P_{2} / P_{1}$ method (for an unkown reason).

Concerning the error in $L^{2}\left(\Gamma_{D}\right)$-norm the value of the stabilization parameter can also be divided into two zones (see Fig. 12, 13 and 14). The first zone where the error decreases when the stabilization parameter $\gamma_{0}$ increases. The second zone, for large values of the parameter, where the error increases (Fig. 13, 14) or remain almost constant (Fig. 12). The Fig. 12 for $P_{1} / P_{0}$ elements indicates that a large value of the stabilization parameter do not affect too much the quality of the solution. This behavior has been noted whenever a piecewise constant multiplier is considered. Conversely, for all remaining couples of elements, an excessive value of the stabilization parameter leads to a bad quality solution (see Fig. 13, 14).

Now, concerning the minimal patch size, the inf-sup condition is proven to be satisfied in [10] for a size equal or greater to $3 h$. Numerically, the inf-sup condition seems to be satisfied for smaller values of the minimal patch size. We found an optimal value between $h$ and $2 h$ in our numerical experiments. For the $P_{1} / P_{0}$ method, a minimal patch size equal to $h$ seems to be inadequate (Fig. 13(a)). A value of $2 h$ is found to be more optimal (Fig. 13(b)). Conversly, a value of $h$ is slightly more optimal for the P1/P1 pair of elements (Fig. 13). 


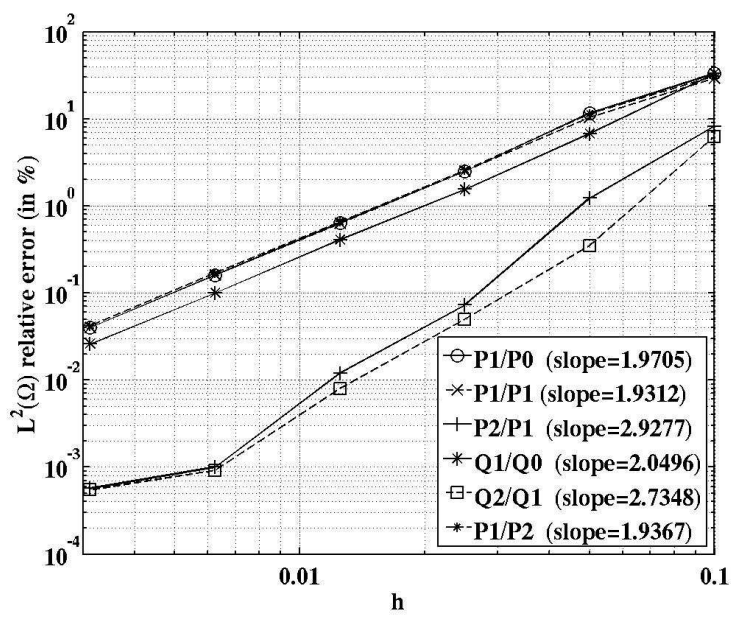

(a) Convergence of $\left\|u-u^{h}\right\|_{0, \Omega}$

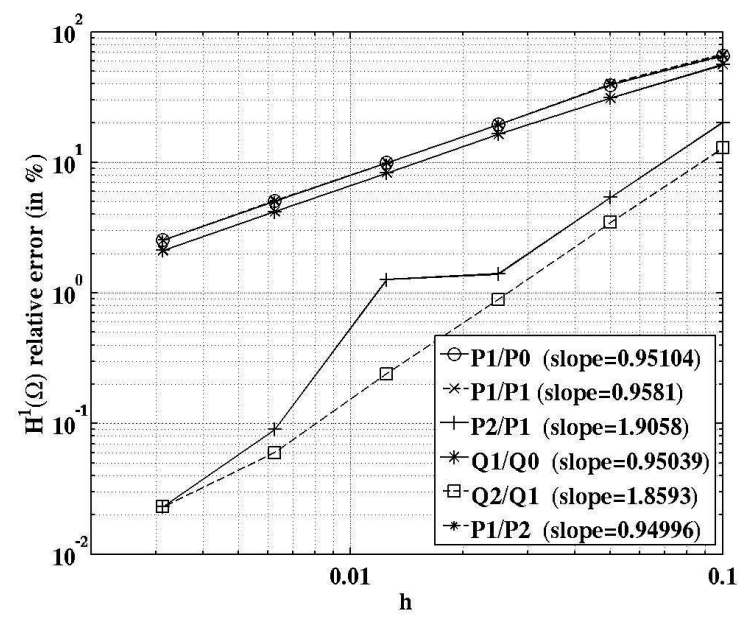

(b) Convergence of $\left\|u-u^{h}\right\|_{1, \Omega}$

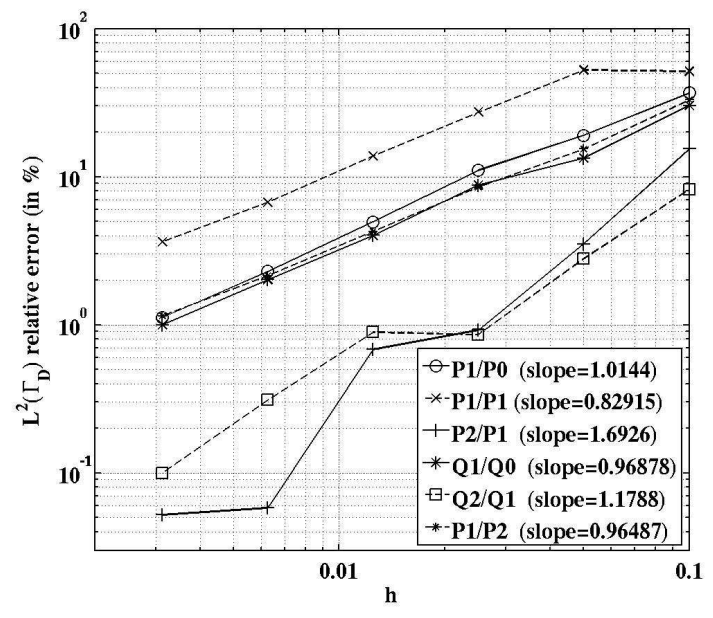

(c) Convergence of $\left\|\lambda^{h}-\lambda\right\|_{0, \Gamma_{D}}$

Figure 11: Convergence curves in the local projection stabilized case.

\subsection{Three-dimensional numerical tests}

In this section, we compare the non-stabilized three-dimensional case to the local projection stabilized three-dimensional case with the following pairs of finite element methods: $P_{2} / P_{1}, P_{1} / P_{1}$, $P_{1} / P_{0} . P_{1} / P_{2}, Q_{2} / Q_{1}$ and $Q_{1} / Q_{0}$.

Without stabilization. Convergence curve in the non-stabilized case are shown in Fig. 15. Perhaps due to the simple chosen geometry and exact solution, no locking phenomenon is observed for the $P_{1} / P_{1}$ and $P_{1} / P_{0}$ methods. However, in these cases, the multiplier do not converge probably due to the presence of spurious modes. The rate of convergence in $H^{1}(\Omega)-$ norm on $u$ is optimal for the $P_{1} / P_{1}, P_{1} / P_{0}, P_{1} / P_{2}$ and $Q_{1} / Q_{0}$ methods (see Fig. 15(b)). For the remaining element $\left(Q_{2} / Q_{1}\right.$ and $\left.P_{2} / P_{1}\right)$ the rate of convergence is limited to $3 / 2$. 


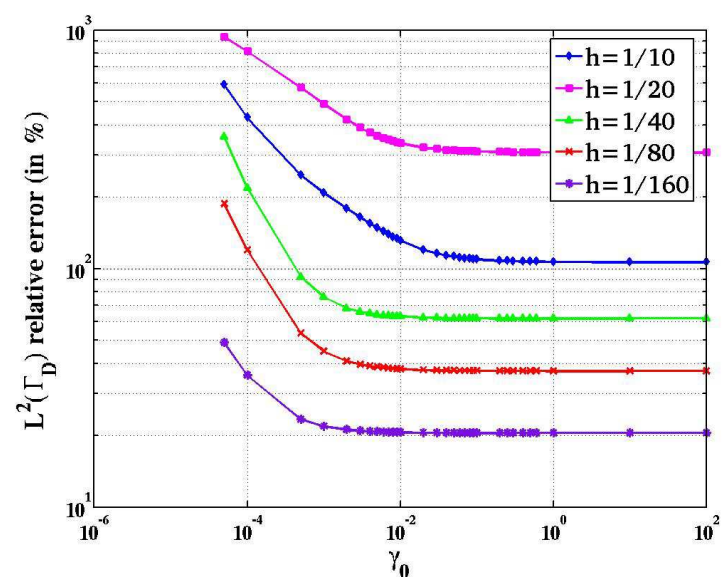

(a) With a minimal patch size equal to $\mathrm{h}$

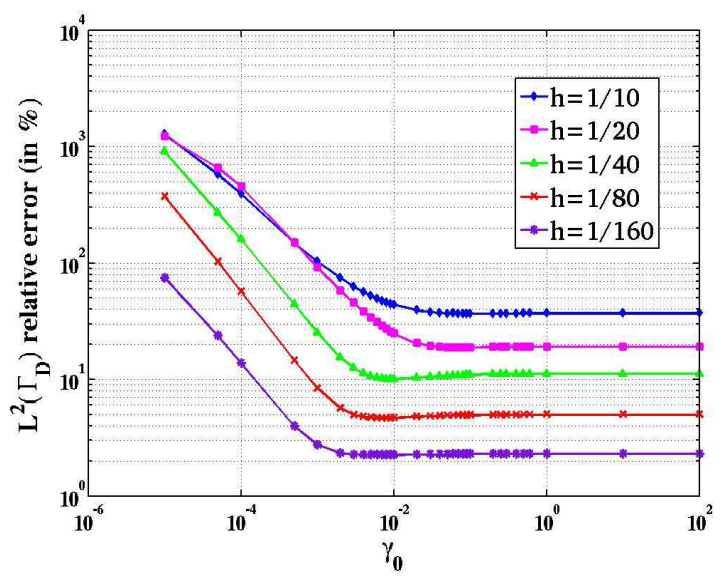

(b) With a minimal patch size equal to $2 \mathrm{~h}$

Figure 12: Influence of the stabilization parameter for the error in in $L^{2}\left(\Gamma_{D}\right)$-norm of the multiplier for the $P_{1} / P_{0}$-elements.

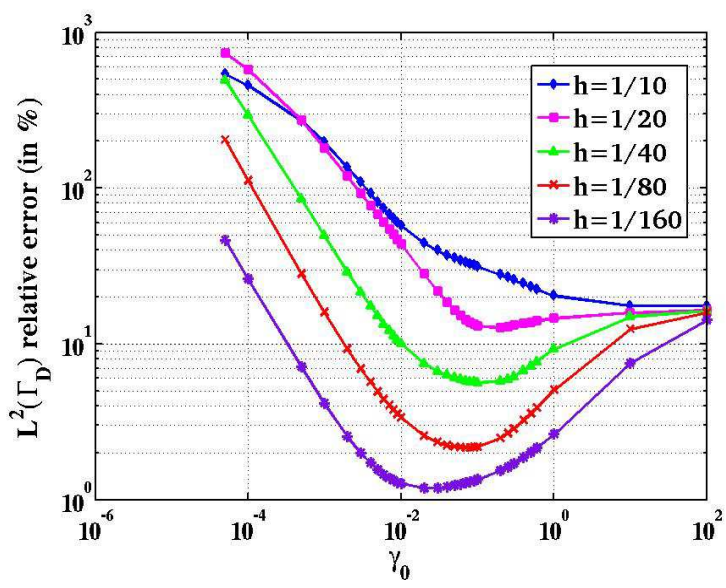

(a) With a minimal patch size equal to $\mathrm{h}$

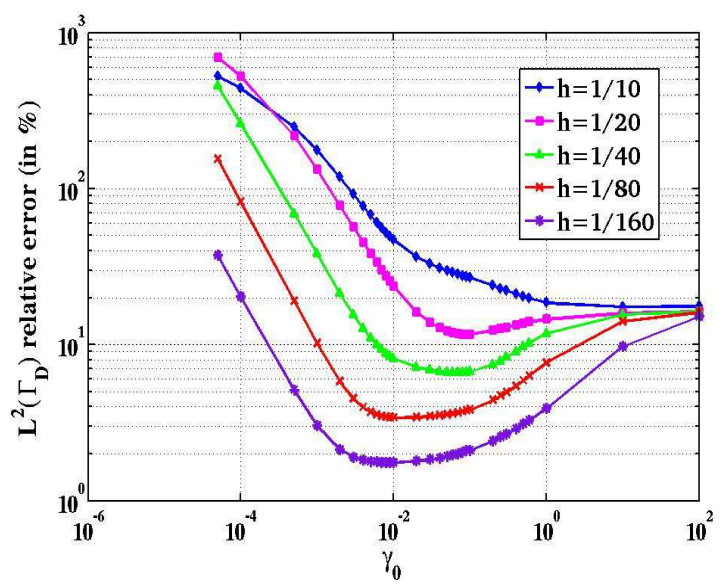

(b) With a minimal patch size equal to $2 \mathrm{~h}$

Figure 13: Influence of the stabilization parameter for the error in $L^{2}\left(\Gamma_{D}\right)$-norm of the multiplier for the $P_{1} / P_{1}$-elements. 


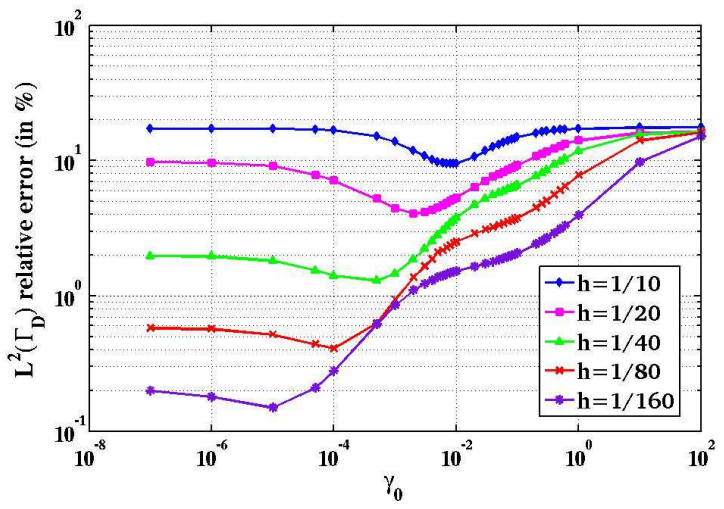

Figure 14: Influence of the stabilization parameter for the error in in $L^{2}\left(\Gamma_{D}\right)$-norm of the multiplier for the $P_{2} / P_{1}$-elements (with a minimal patch size equal to $\mathrm{h}$ ).

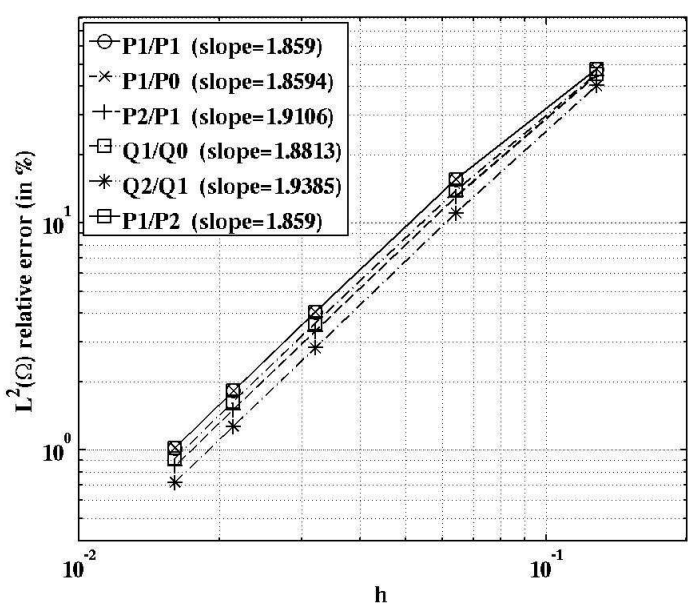

(a) Convergence of $\left\|u-u^{h}\right\|_{0, \Omega}$

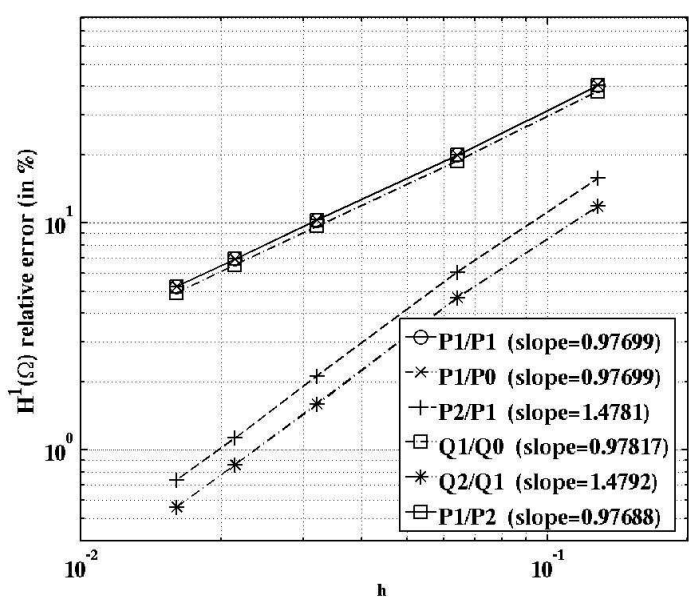

(b) Convergence of $\left\|u-u^{h}\right\|_{1, \Omega}$

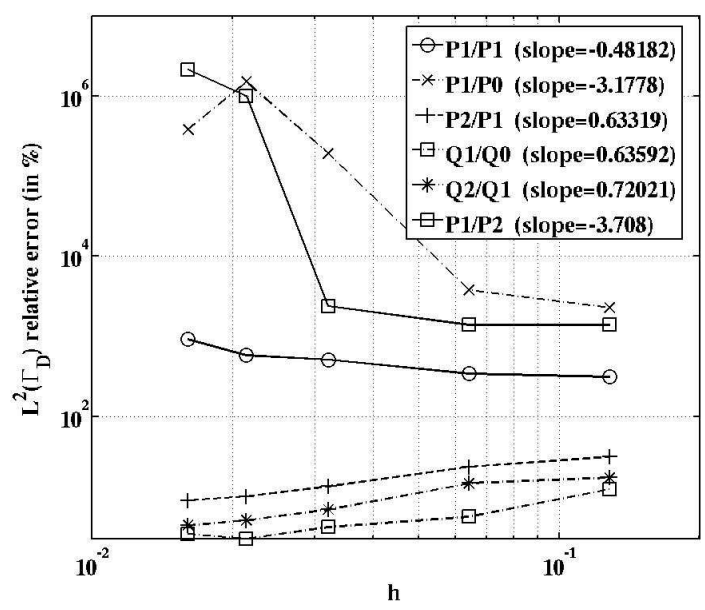

(c) Convergence of $\left\|\lambda^{h}-\lambda\right\|_{0, \Gamma_{D}}$

Figure 15: Convergence curves in the three-dimensional non-stabilized case. 
Local projection stabilization. The local projection stabilization gives an optimal rate of convergence for all pairs of elements and eliminates the spurious modes for the $P_{1} / P_{1}, P_{1} / P_{0}$ and $P_{1} / P_{2}$ methods. Especially, the rate of convergence in $H^{1}(\Omega)$-norm for the $Q_{2} / Q_{1}$ and $P_{2} / P_{1}$ are improved compared to the non-stabilized case.

Except for the $Q_{2} / Q_{1}$ pair of elements, the convergence rate for the $L^{2}\left(\Gamma_{D}\right)$-norm for the multiplier are optimal (more than 1.5). For the $Q_{2} / Q_{1}$ pair of elements the convergence rate for the $L^{2}\left(\Gamma_{D}\right)$-norm is optimal but limiter to 1.1 for an unknown reason. The rate of convergence in $L^{2}(\Omega)$-norm is limited to 2 for all the methods. For quadratic methods, this may be due to the use of level set function of order 1 to approximate the curved domain witch limited theoretically the rate of convergence to $3 / 2$.

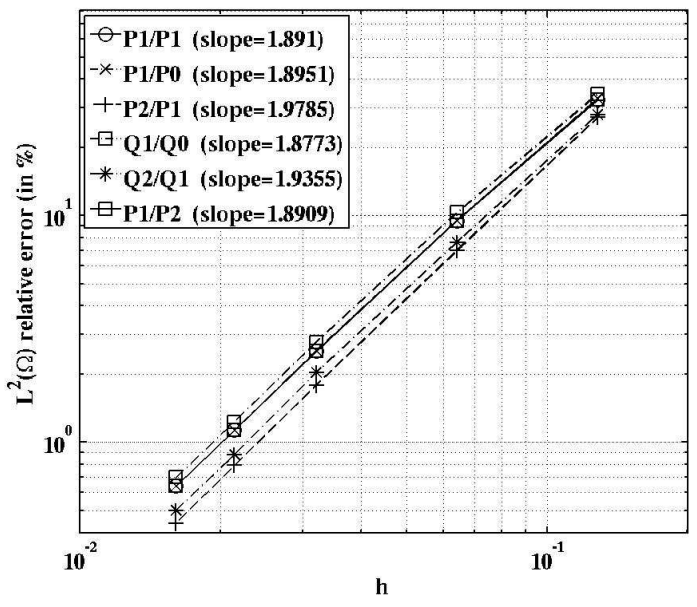

(a) Convergence of $\left\|u-u^{h}\right\|_{0, \Omega}$

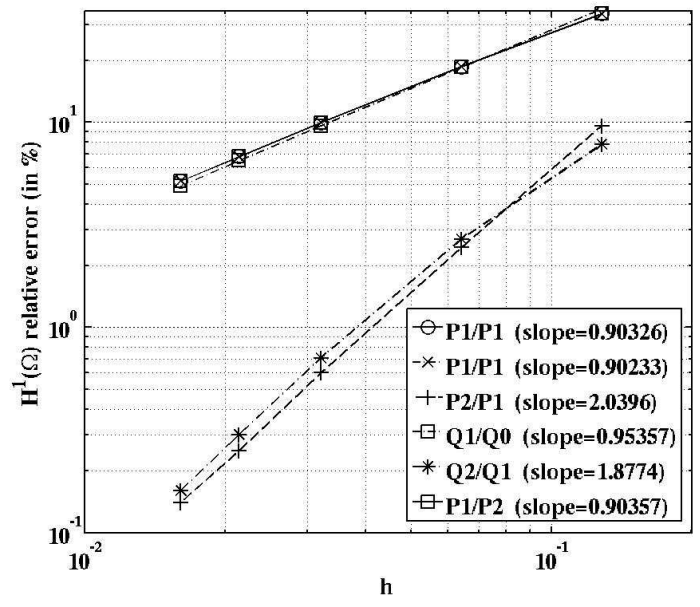

(b) Convergence of $\left\|u-u^{h}\right\|_{1, \Omega}$

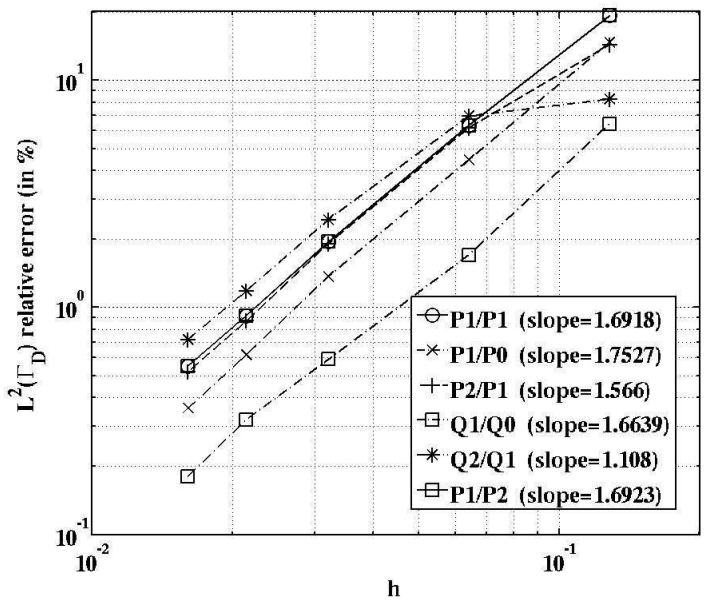

(c) Convergence of $\left\|\lambda^{h}-\lambda\right\|_{0, \Gamma_{D}}$

Figure 16: Convergence curves in the three-dimensional local projection stabilized case.

\section{Concluding remarks}

In this paper, we presented a new stabilization technique based on local projections for the fictitious domain method inspired by the Xfem introduced in $[8,13]$. 
A main advantage compared to some other stabilization techniques like the Barbosa-Hughes one is that is only affects the multiplier equation in a manner that is independent of the problem to be solved. This makes the extension to other linear or nonlinear problems very easy.

The two-dimensional theoretical result do not ensure an optimal rate of convergence when a quadratic finite element is used for the main unknown due to the fact that the local projection is made on piecewise constants. The method could be generalized to the projection on (discontinuous) piecewise affine or piecewise quadratic functions for high order approximations.

The extension to the three-dimensional case of the theoretical result is of course subject to obtaining an inf-sup condition of the same kind of the one obtained in [10].

\section{References}

[1] S. Amdouni, P. Hild, V. Lleras, M. Moakher, Y. Renard. A stabilized Lagrange multiplier method for the enriched finite-element approximation of contact problems of cracked elastic bodies, ESAIM: M2AN, 46(2012): 813-839.

[2] Ph. Angot, Ch.-H. Bruneau, and P. Fabrie. A penalization method to take into account obstacles in incompressible viscous flows. Nümerische Mathematik, 81(4) (1999):497-520.

[3] É. Béchet, N. Moës, B. Wohlmuth. A stable Lagrange multiplier space for stiff interface conditions within the extended finite element method. Int. J. Numer. Meth. Engng. 78:8(2009), 931-954.

[4] H. J.C. Barbosa and T.J.R. Hughes, The finite element method with Lagrange multipliers on the boundary: circumventing the Babuška-Brezzi condition, Comput. Methods Appl. Mech. Engrg., 85 (1991), pp. 109-128.

[5] H. J.C. Barbosa and T.J.R. Hughes, Boundary Lagrange multipliers in finite element methods: error analysis in natural norms, Numer. Math., 62 (1992):1-15.

[6] S. Bertoluzza, M. Ismail, and B. Maury. The FBM method : Semi-discrete scheme and some numerical experiments. Lecture Notes in Comp. Sc. and Eng., 2004.

[7] E. Burman and P. Hansbo. Fictitious domain finite element methods using cut elements:I. A satabilized Lagrange multiplier method. Comput. Meth. Appl. Mech. Engrg. 199:2680-2686, 2010 .

[8] E. Burman and P. Hansbo. Fictitious domain finite element methods using cut elements:II. A satabilized Nitsche method. Applied Numerical Mathematics 62(4):328-341, 2012.

[9] E.J. Dean, Q.V. Dinh, R. Glowinski, J. He, T.W. Pan, and J. Périaux. Least squares/domain imbedding methods for Neumann problems : application to fluid dynamics. Domain Decomposition Methods for Partial Differential Equations, D. E. Keyes, T. F. Chan, G. Meurant, J. S. Scroggs, R. G. Voigt eds., SIAM, Philadelphia, pp. 451-475, 1992.

[10] V. Girault and R. Glowinski. Error analysis of Fictitious Domain Method Applied to Dirichlet Problem. Japan J. Indsut. Appl. Math. 12:487-514, 1995.

[11] R. Glowinski and Y. Kuznetsov. On the solution of the Dirichlet problem for linear elliptic operators by a distributed Lagrange multiplier method. C.R. Acad. Sci. Paris, t.327, Serie I, pp. 693-698, 1998. 
[12] R. Glowinski, T.W. Pan, Jr.R. Wells, and X. Zhou. Wavelet and finite element solutions for the Neumann problem using fictitious domains. Journal Comput. Phys., 126(1) (1996):40-51.

[13] J. Haslinger and Y. Renard. A new fictitious domain approach inspired by the extended finite element method. J. Numer. Anal. 47(2):1474-1499, 2009.

[14] G. Karypis and V. Kumar. MeTis: Unstructured Graph Partitioning and Sparse Matrix Ordering System. http://www.cs.umn.edu/ metis, 2009, University of Minnesota, Minneapolis, MN.

[15] G.I. Marchuk. Methods of Numerical Mathematics. Application of Math. 2, Springer-Verlag New York (1rst ed. 1975), 1982.

[16] B. Maury. A Fat Boundary Method for the Poisson problem in a domain with holes. SIAM, J. of Sci. Comput., 16(3) (2001):319-339.

[17] N. Mö̈s, E. BÉchet, And M. Tourbier. Imposing Dirichlet boundary conditions in the eXtended Finite Element Method. Int. J. Numer. Meth. Engng., 67, 12 (2006), 354-381.

[18] N. Moës, J. Dolbow, and T. Belytschko. A finite element method for crack growth without remeshing. Int. J. Numer. Meth. Engng., 46:131-150, 1999.

[19] J. Nitsche, Über ein Variationsprinzip zur Lösung Dirichlet-Problemen bei Verwendung von Teilräumen, die keinen Randbedingungen unterworfen sind, Abh. Math. Univ. Hamburg, 36 (1971), pp. 9-15.

[20] C.S. Peskin. Flow patterns around heart valves: A numerical method. J. Comput. Phys. 10(2) (1972):252-271.

[21] C.S. Peskin. The immersed boundary method. Acta Numerica, 11 (2002):479-517.

[22] J. Pommier and Y. Renard Getfem ++ . An open source generic $C++$ library for finite element methods, http://download.gna.org/getfem/html/homepage.

[23] L.A. Rukhovets. A remark on the method of fictive domains. Differential Equations,3,4 (in Russian), 1967.

[24] V.K. Saul'ev. On the solution of some boundary value problems on high performance computers by fictitious domain method. Siberian Math. Journal, 4:4(1963):912-925 (in Russian).

[25] R. Stenberg, On some techniques for approximating boundary conditions in the finite element method, J. Comput. Appl. Math., 63 (1995), pp. 139-148.

[26] N. Sukumar, D. L. Chopp, N. Moës and T. Belytschko. Modeling holes and inclusions by level sets in the extended finite element method. Comput. Meth. Appl. Mech. Eng., 90(46, 47):6183-6200, 2001. 